\title{
Alphasatellitidae: a new family with two subfamilies for the classification of geminivirus- and nanovirus-associated alphasatellites
}

\author{
Rob W. Briddon ${ }^{1}$. Darren P. Martin ${ }^{2}$. Philippe Roumagnac ${ }^{3,4}$. Jesús Navas-Castillo ${ }^{5}$ Elvira Fiallo-Olivé ${ }^{5}$. \\ Enrique Moriones ${ }^{5} \cdot$ Jean-Michel Lett ${ }^{6} \cdot$ F. Murilo Zerbini ${ }^{7} \cdot$ Arvind Varsani $^{8,9}$
}

Received: 26 March 2018 / Accepted: 25 April 2018 / Published online: 9 May 2018

(c) Springer-Verlag GmbH Austria, part of Springer Nature 2018

\begin{abstract}
Nanoviruses and geminiviruses are circular, single stranded DNA viruses that infect many plant species around the world. Nanoviruses and certain geminiviruses that belong to the Begomovirus and Mastrevirus genera are associated with additional circular, single stranded DNA molecules ( 1-1.4 kb) that encode a replication-associated protein (Rep). These Rep-encoding satellite molecules are commonly referred to as alphasatellites and here we communicate the establishment of the family Alphasatellitidae to which these have been assigned. Within the Alphasatellitidae family two subfamilies, Geminialphasatellitinae and Nanoalphasatellitinae, have been established to respectively accommodate the geminivirusand nanovirus-associated alphasatellites. Whereas the pairwise nucleotide sequence identity distribution of all the known geminialphasatellites $(\mathrm{n}=628)$ displayed a troughs at $\sim 70 \%$ and $88 \%$ pairwise identity, that of the known nanoalphasatellites $(\mathrm{n}=54)$ had a troughs at $\sim 67 \%$ and $\sim 80 \%$ pairwise identity. We use these pairwise identity values as thresholds together with phylogenetic analyses to establish four genera and 43 species of geminialphasatellites and seven genera and 19 species of nanoalphasatellites. Furthermore, a divergent alphasatellite associated with coconut foliar decay disease is assigned to a species but not a subfamily as it likely represents a new alphasatellite subfamily that could be established once other closely related molecules are discovered.
\end{abstract}

\section{Introduction}

The family Geminiviridae consists of viruses with either monopartite or bipartite circular single-stranded (ss)DNA genomes, with each component individually encapsidated

Handling Editor: Sead Sabanadzovic.

Disclaimer A.V. is an elected member of the ICTV EC. F.M.Z. is the chair of the Plant Virus Subcommittee of the ICTV. R.W.B., D.P.M., P.R., J.N-C., E.F-O., J-M.L., F.M.Z. and A.V. are current members of the Geminiviridae and Tolecusatellitidae Study Group of the ICTV.

Electronic supplementary material The online version of this article (https://doi.org/10.1007/s00705-018-3854-2) contains supplementary material, which is available to authorized users.

Rob W. Briddon

rob.briddon@gmail.com

Arvind Varsani

arvind.varsani@asu.edu

Extended author information available on the last page of the article in twinned quasi-isometric (geminate) $22-38 \mathrm{~nm}$ virions [3, $17,55]$. The family contains nine genera $[46,48,54]$, of which two are relevant to this communication here - Begomovirus and Mastrevirus. Begomoviruses and mastreviruses transmitted by the whitefly Bemisia tabaci and by leafhoppers, respectively, in a circulative manner. The genomes/ genomic components of geminiviruses are typically $2600-$ 3600 nucleotides [5, 54].

The family Nanoviridae encompasses viruses with multicomponent circular ssDNA genomes that are individually encapsidated within isometric $17-20 \mathrm{~nm}$ virions. The family is divided into two genera, Nanovirus and Babuvirus, containing viruses transmitted by aphids in a circulative, nonpropagative manner [50,51]. Members of the genus Nanovirus infect dicotyledonous host plants whereas those of the genus Babuvirus infect monocotyledonous plants. Typically, the genomes of nanoviruses have eight components and the genomes of babuviruses consist of six components. The genomic components of viruses in both genera typically range in size from 970 to 1116 nucleotides [51]. The bona fide genome components of a nanovirus genome share two 
regions of sequence similarity known as the common region stem-loop (CR-SL) and the common region major (CRM; Figure 1). The DNA-R component of nanoviruses encodes a replication-associated protein (Rep) $[7,12,13,15,16,25$, $38,42]$ which is involved in replicating all bona fide genome components.

Viruses of the family Nanoviridae, and some begomoviruses and mastreviruses in the family Geminiviridae may be associated with additional circular ssDNA components that resemble the DNA-R components of nanoviruses; these will hereinafter be referred to here as alphasatellites. In common with the DNA-R component of nanoviruses, the alphasatellites encode a Rep protein and have a predicted stem-loop structure with, in most cases, the nonanucleotide sequence TAGTATTAC forming part of the loop (Figure 1). Alphasatellites associated with nanoviruses have a similar size to that of typical nanovirus components ( 1100 nucleotides), lack the CR-SL and CRM of their helper viruses and are also unable to trans-replicate the bona fide genome components of these helper viruses [18, 43-45]. Alphasatellites have also been identified in association with begomoviruses and, in one reported case, a mastrevirus [4, 24]. Typically comprising 1400 nucleotides, the geminivirus associated alphasatellites are significantly larger than both nanovirus components and nanovirus alphasatellites [4]. This size difference, primarily due to the presence in geminivirus alphasatellites of an adenine rich region of sequence and a larger rep gene, is thought to be necessary for the encapsidation of geminivirus alphasatellites by their helper viruses. Geminiviruses have strict restrictions on the size of circular ssDNA molecule that they can encapsidate being half the geminivirus genome length ( 1400 nucleotides), geminivirus alphasatellites could possibly be encapsidated in isometric (half geminate) virions [8]. For both nanovirus- and geminivirusassociated alphasatellites the sequence relatedness between, and similarity in structure to, nanovirus DNA-R components has led to the hypothesis that the satellites evolved from DNA-R components that were "captured" following coinfections [26, 40].

It is interesting to note that most geminivirus-associated alphasatellites described from the Old World have been associated with monopartite begomoviruses infections that frequently include the presence of betasatellites $[39,52,56]$. In the New World where monopartite begomoviruses are very rare, betasatellites have not been identified and alphasatellites have only ever been reported to occur in association with bipartite Begomovirus infections [10, 32, 37].

The precise impact of alphasatellites on begomovirus and nanovirus infections remains unclear. The presence of nanoalphasatellites in some nanovirus infections has been associated with reduced nanovirus infectivity [45] while geminialphasatellites have been associated with reduced or exacerbated symptoms [27, 32] and/or reduced titer of genomic or betasatellite DNA [19, 21]. Whereas the encoded Rep proteins of alphasatellites associated with the begomoviruses Gossypium darwinii symptomless virus and Gossypium mustelinium symptomless virus suppress post-transcriptional gene silencing [30], those encoded by various other begomovirus alphasatellites are suppressors of transcriptional gene silencing [1]. Due to the rising number of alphasatellites being described, owing mainly to both increasing interest in begomoviruses and nanoviruses, and the ease with which these molecules can be isolated and characterized, there is an urgent need for a robust and workable system of nomenclature and classification for these molecules. Since alphasatellites rely on a helper virus for their spread and are therefore not independent entities, there is very little biological data that can be unequivocally attributed to any of these molecules. It is therefore necessary to base an alphasatellite classification system primarily

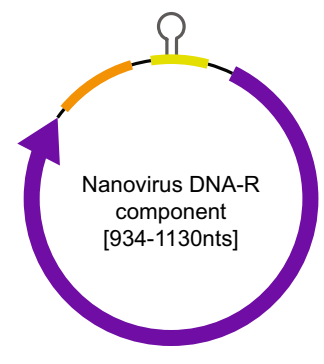

Nanoviridae

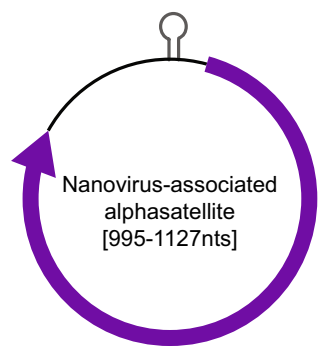

Nanoalphasatellitinae

Alphasatellitidae
Fig. 1 Illustration of the Rep-encoding DNA molecules of nanovirus- and geminivirus-associated alphasatellites in comparison to the DNA-R components of nanoviruses. The diagrams include the position of the replication-associated protein (Rep), the TATA box of the presumed Rep promoter, an adenine rich sequence (A-rich) in geminivirus-associated alphasatellites and the common region major

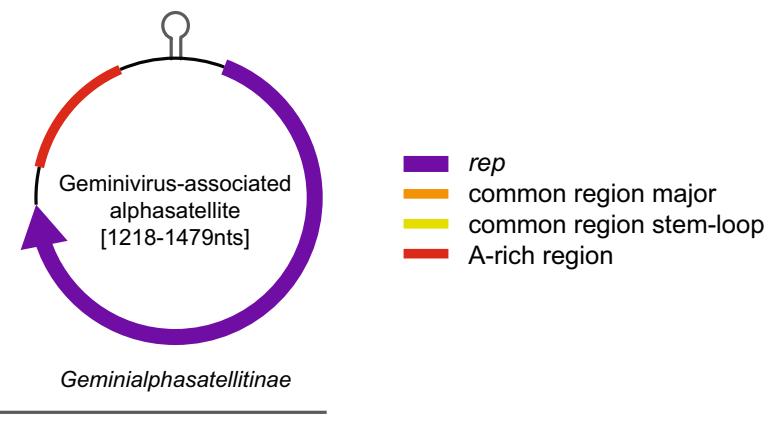

and common region stem-loop of nanovirus DNA-R components. All components have a predicted stem-loop structure (at position zero) with, in most cases, the nonanucleotide sequence TAGTATTAC forming part of the loop. This likely forms the origin of virion-strand DNA replication that is nicked by Rep to initiate rolling-circle replication 
on information extracted from the nucleotide sequences of these molecules. Such a system could then be adjusted once biological evidence becomes available.

We communicate the creation of the family Alphasatellitidae to which alphasatellites have been assigned and the establishment of two subfamilies based on the fact that there are clear differences between geminivirus- and nanovirusassociated alphasatellites. These include genome length and a distinctive A-rich region downstream of the Rep coding region of the alphasatellites that are associated with geminiviruses. The two sub families are

1) Geminialphasatellitinae: geminivirus-associated alphasatellites

2) Nanoalphasatellitinae: nanovirus-associated alphasatellites

\section{Geminialphasatellitinae}

Sequences of geminivirus-associated alphasatellites were downloaded from GenBank ( $1^{\text {st }}$ May 2017). These were checked to ensure they were complete molecules, had intact Rep open reading frames and were not sub-genomic begomovirus genome components. The filtered 628 geminivirus-associated alphasatellites were analysed with SDT v1.2 [29]. The 628 sequences that remained were aligned using MUSCLE [9] and used to infer a maximum likelihood phylogenetic tree using IQ-TREE [31] with the nucleotide substitution model, GTR $+\mathrm{I}+\mathrm{G} 4$ (adjudged to be the best fitting by ModelFinder [20]). This tree was rooted with the representative DNA-R sequence of nanoviruses (AB027511, AF102780, AJ290434, AJ749894, EF546807, GU553134, HE654123, JX867550, KC978949, KC979035,
KC979054, KX534388, KX534389, KX534390, KX534391, MF535450)

The distribution of the 197506 pairwise identities between each of the possible pair of these 628 sequences had troughs at $\sim 70 \%$ and $\sim 88 \%$ pairwise identity (Figure 2 ) indicating that these values could respectively ben used as genus and species demarcation thresholds, respectively, thus yielding a classification of these sequences with a minimal degree of conflict (Figure 2). These demarcation thresholds are broadly consistent with the viruses in the different species and genera having distinctive helper viruses, geographical distributions and phylogenetic placements (Figure 3; Figure 4).

The name Alphasatellitidae derives from the name alphasatellite which is now in common usage for this group of satellites. Upon first identification, the begomovirus-associated alphasatellites were called DNA 1 in recognition of their relatedness to the Rep-encoding components of nanoviruses (DNA-R) which at the time were called DNA 1 [26]. The alphasatellite genera names that we have chosen are derived from the names of the type species so, for example, the genus name Colecusatellite derives from the name of the first begomovirus alphasatellite identified, Cotton leaf curl Multan alphasatellite. The alphasatellite species names are, whenever possible, derived from the name of the helper virus with which they were first identified; so cotton leaf curl Multan alphasatellite was first identified in a cotton plant infected with cotton leaf curl Multan virus.

Based on the above criteria, four genera and 43 species are established in the subfamily Geminialphasatellitinae. The classification of the genera and species in the subfamily Geminialphasatellitinae is summarized in Table 1.

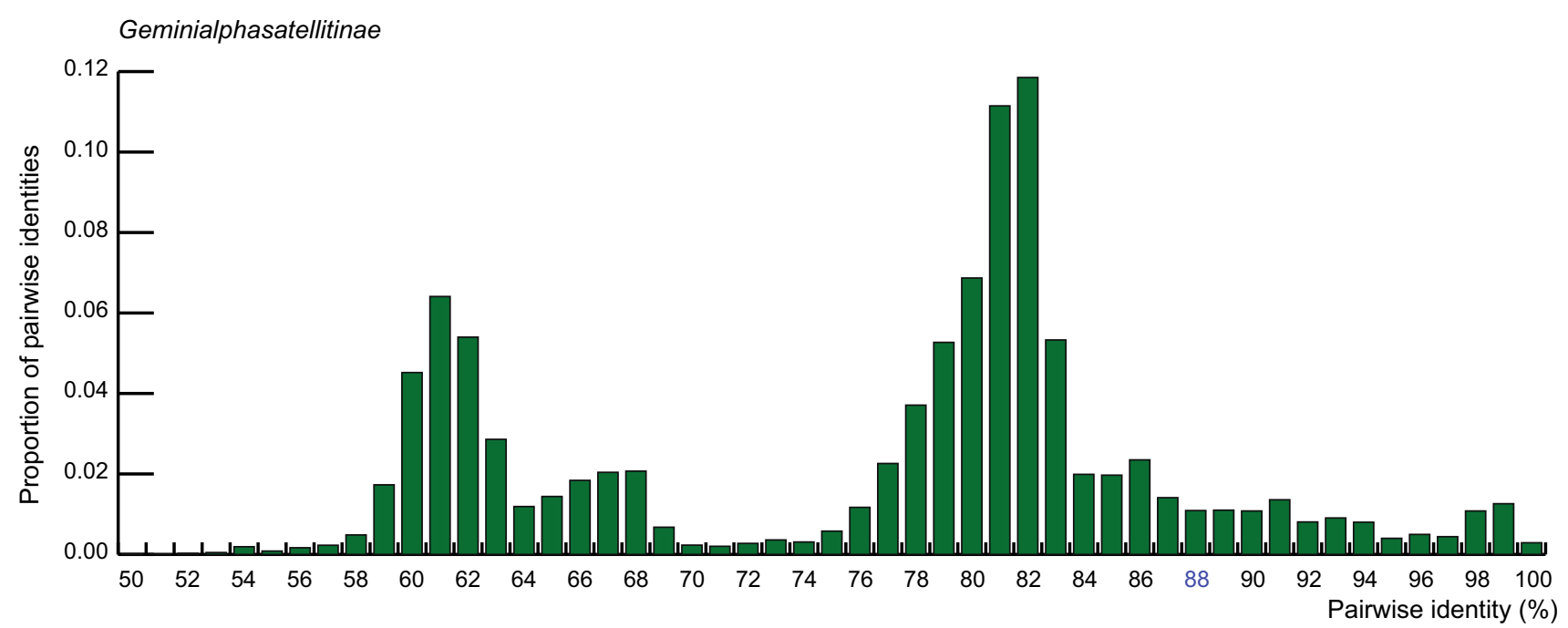

Fig. 2 Distribution of pairwise identities of geminivirus-associated alphasatellites determined using SDT v1.2 [29] 
Colecusatellite

Gosmusatellite

Ageyesisatellite

Clecrusatellite

Unassigned

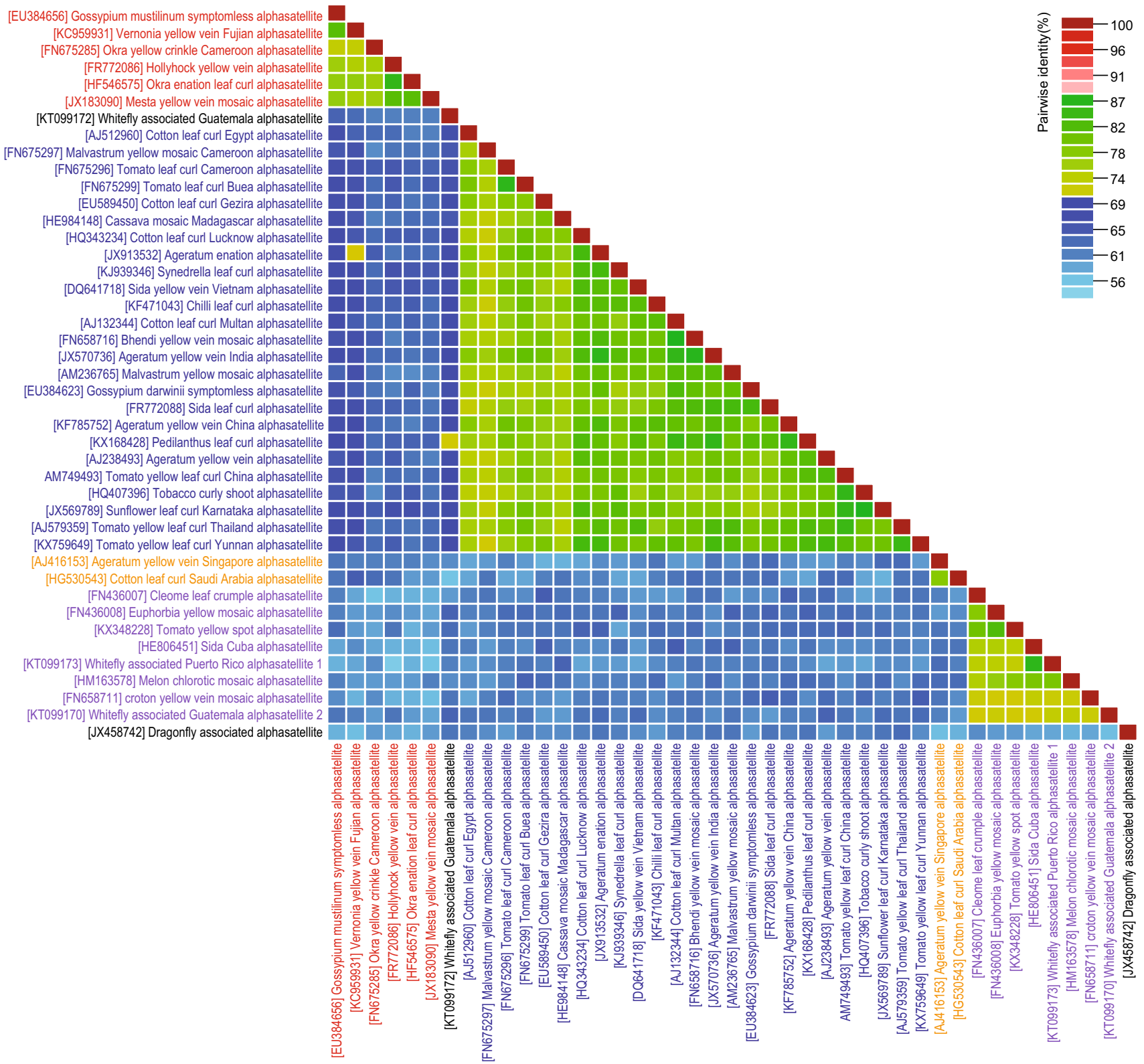

Fig. 3 A 'three color' pairwise identity matrix inferred using SDT v1.2 [29] showing that both the genera demarcation threshold of 70\% and that for species at $88 \%$ are supported

\section{The 43 Geminialphasatellitinae species}

1. All have a distinctive organization consisting of a single conserved Rep encoding gene in the virion-sense, and a predicted hairpin structure at their presumed origin of virion strand replication that contains, in most cases, a TAGTATTAC loop sequence.

2. With the exception of two alphasatellites, all have been shown to be associated with a geminivirus. 


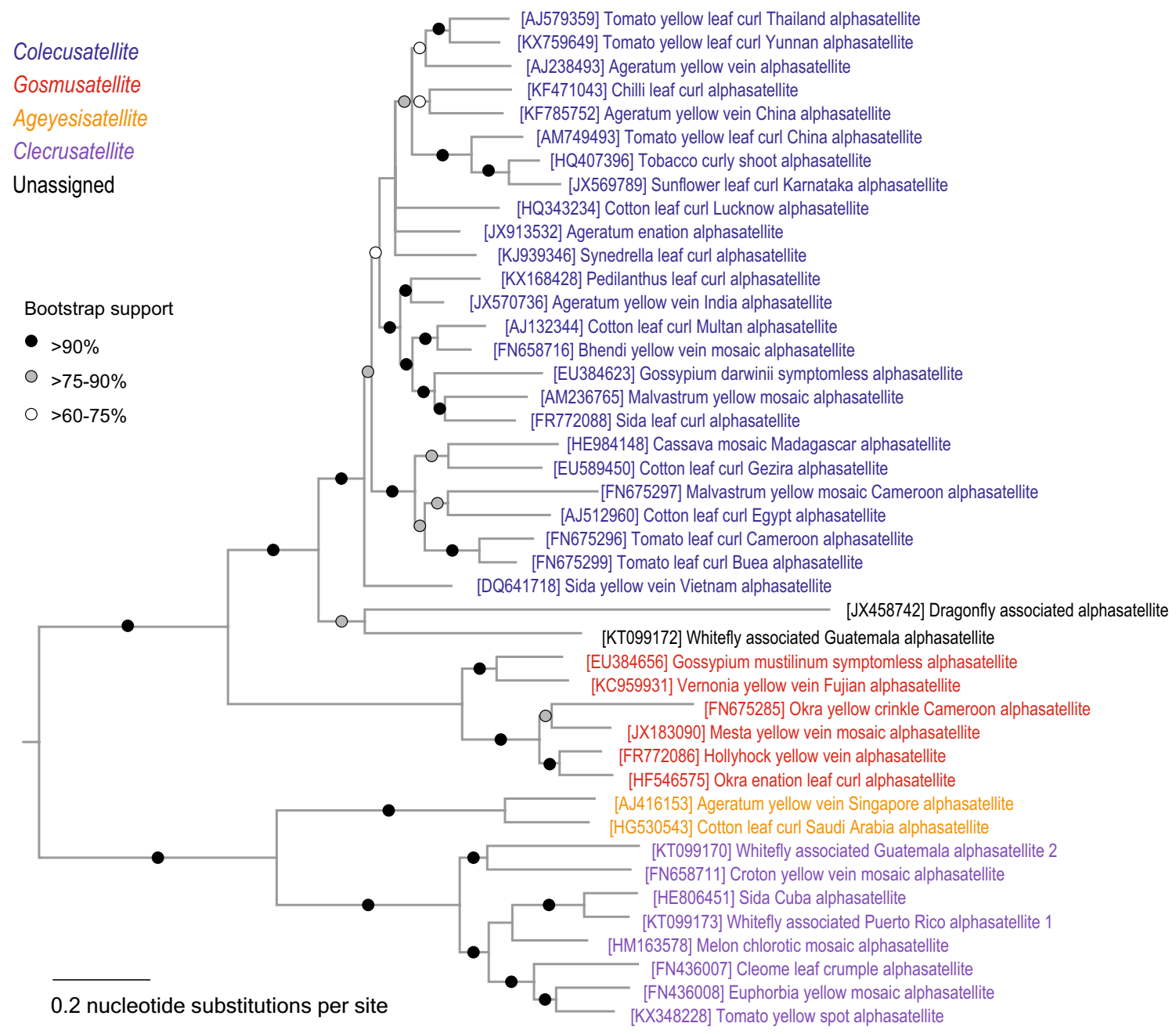

Fig. 4 Maximum likelihood phylogenetic tree of representative geminivirus-associated alphasatellite sequences from each species inferred using IQ-TREE [31] with GTR+I+G4 chosen as the best-fit model. Branches with less than $60 \%$ bootstrap support have been collapsed

For two alphasatellite sequences that were included in the analysis no helper begomovirus is known. Sequence JX458742 (species Dragonfly associated alphasatellite) was isolated from a dragonfly caught in Puerto Rico and no virus could conclusively be shown to be associated with the molecule. Sequence KT099172 (species Whitefly associated Guatemala alphasatellite 1) was isolated from a B. tabaci whitefly originating from Guatemala and no virus could conclusively be shown to be associated with the molecule.

\section{Guidelines for classification of new geminialphasatellites species}

Pairs of complete geminialphasatellite sequences with $\geq 88 \%$ pairwise identity calculated using pairwise MUSCLE alignments with similarities calculated ignoring sites with gaps (such as is implemented in SDT v1.2 [29]), should be considered as members of the same species. Those that have
$<88 \%$ pairwise identity can be considered as new species when coupled with good phylogenetic support.

We recommend that the following steps, similar to those outlined for geminiviruses $[6,28,46]$ be taken to resolve such conflicts in cases where 1) a complete geminialphasatellite sequence shares $\geq 88 \%$ pairwise identity to sequences that have been assigned to two different species; or 2) a complete geminialphasatellite sequence shares $\geq 88 \%$ genomewide pairwise identity to one or a few sequences assigned to a particular species, even though it shares $<88 \%$ identity with the majority of sequences in that species

1) The new geminialphasatellite sequence should be considered as belonging to the species containing sequences with which it shares the highest percentage pairwise identity.

2) The geminialphasatellite should be classified as belonging to any species with which it shares $\geq 88 \%$ pairwise 
Table 1 Summary of the genera and species in subfamilies Geminialphasatellitinae and Nanoalphasatellitinae

\begin{tabular}{|c|c|c|c|c|c|c|c|}
\hline Subfamily & Genus & & Species & Alphasatellite & Accession & $\begin{array}{l}\text { Alphasatellite } \\
\text { acronym }\end{array}$ & Isolate ID \\
\hline \multirow[t]{21}{*}{$\begin{array}{l}\text { Geminialphasatel- } \\
\text { litinae }\end{array}$} & \multirow[t]{2}{*}{ Ageyesisatellite } & \multirow[t]{2}{*}{$\mathbf{\square}$} & $\begin{array}{l}\text { Ageratum yellow vein } \\
\text { Singapore alphas- } \\
\text { atellite }\end{array}$ & $\begin{array}{l}\text { ageratum yellow } \\
\text { vein Singapore } \\
\text { alphasatellite }\end{array}$ & AJ416153 & AYVSGA & SG-98 \\
\hline & & & $\begin{array}{l}\text { Cotton leaf curl Saudi } \\
\text { Arabia alphasatellite }\end{array}$ & $\begin{array}{l}\text { cotton leaf curl } \\
\text { Saudi Arabia alph- } \\
\text { asatellite }\end{array}$ & HG530543 & CLCuSAA & SA-Jazan-13 \\
\hline & \multirow[t]{8}{*}{ Clecrusatellite } & \multirow[t]{8}{*}{$\mathbf{\square}$} & $\begin{array}{l}\text { Cleome leaf crumple } \\
\text { alphasatellite }\end{array}$ & $\begin{array}{l}\text { cleome leaf crumple } \\
\text { alphasatellite }\end{array}$ & FN436007 & CILCrA & $\begin{array}{l}\text { BR-Mato Grosso do } \\
\text { Sul-07 }\end{array}$ \\
\hline & & & $\begin{array}{l}\text { Croton yellow vein } \\
\text { mosaic alphasatel- } \\
\text { lite }\end{array}$ & $\begin{array}{l}\text { croton yellow vein } \\
\text { mosaic alphasat- } \\
\text { ellite }\end{array}$ & FN658711 & CrYVMA & $\begin{array}{l}\text { N-Haryana-Acaly- } \\
\text { pha-07 }\end{array}$ \\
\hline & & & $\begin{array}{l}\text { Euphorbia yellow } \\
\text { mosaic alphasatel- } \\
\text { lite }\end{array}$ & $\begin{array}{l}\text { euphorbia yellow } \\
\text { mosaic alphasat- } \\
\text { ellite }\end{array}$ & FN436008 & EuYMA & $\begin{array}{l}\text { BRl-Mato grosso do } \\
\text { sul-1-07 }\end{array}$ \\
\hline & & & $\begin{array}{l}\text { Melon chlorotic } \\
\text { mosaic alphasatel- } \\
\text { lite }\end{array}$ & $\begin{array}{l}\text { melon chlorotic } \\
\text { mosaic alphasat- } \\
\text { ellite }\end{array}$ & HM163578 & MeCMA & $\begin{array}{l}\text { VE-2009_02_04_0- } \\
09\end{array}$ \\
\hline & & & $\begin{array}{l}\text { Sida Cuba alphasat- } \\
\text { ellite }\end{array}$ & $\begin{array}{l}\text { sida Cuba alphas- } \\
\text { atellite }\end{array}$ & HE806451 & SiCUA & CU-Trinidad-07 \\
\hline & & & $\begin{array}{l}\text { Tomato yellow spot } \\
\text { alphasatellite }\end{array}$ & $\begin{array}{l}\text { tomato yellow spot } \\
\text { alphasatellite }\end{array}$ & KX348228 & ToYSA & BR-Dou1095.1-11 \\
\hline & & & $\begin{array}{l}\text { Whitefly associated } \\
\text { Guatemala alphasat- } \\
\text { ellite } 2\end{array}$ & $\begin{array}{l}\text { whitefly associated } \\
\text { Guatemala alphas- } \\
\text { atellite } 2\end{array}$ & КT099170 & WfaGA 2 & GT-GtTo2_1-10 \\
\hline & & & $\begin{array}{l}\text { Whitefly associated } \\
\text { Puerto Rico alphas- } \\
\text { atellite } 1\end{array}$ & $\begin{array}{l}\text { whitefly associ- } \\
\text { ated Puerto Rico } \\
\text { alphasatellite } 1\end{array}$ & КT099173 & WfaPRA 1 & PR-PR3_6-10 \\
\hline & \multirow[t]{11}{*}{ Colecusatellite } & & $\begin{array}{l}\text { Ageratum enation } \\
\text { alphasatellite }\end{array}$ & $\begin{array}{l}\text { ageratum enation } \\
\text { alphasatellite }\end{array}$ & JX913532 & AEA & IN-Luc-12 \\
\hline & & & $\begin{array}{l}\text { Ageratum yellow vein } \\
\text { alphasatellite }\end{array}$ & $\begin{array}{l}\text { ageratum yellow } \\
\text { vein alphasatellite }\end{array}$ & AJ238493 & AYVA & SG-98 \\
\hline & & & $\begin{array}{l}\text { Ageratum yellow vein } \\
\text { China alphasatellite }\end{array}$ & $\begin{array}{l}\text { ageratum yellow } \\
\text { vein China alphas- } \\
\text { atellite }\end{array}$ & KF785752 & AYVCNA & $\begin{array}{r}\text { PH-Davao-SN3-Syn- } \\
\text { edrella nodiflora-12 }\end{array}$ \\
\hline & & & $\begin{array}{l}\text { Ageratum yellow vein } \\
\text { India alphasatellite }\end{array}$ & $\begin{array}{l}\text { ageratum yellow } \\
\text { vein India alphas- } \\
\text { atellite }\end{array}$ & JX570736 & AYVIA & $\begin{array}{l}\text { IN-Luc-parthe- } \\
\text { nium-12 }\end{array}$ \\
\hline & & & $\begin{array}{l}\text { Bhendi yellow vein } \\
\text { alphasatellite }\end{array}$ & $\begin{array}{l}\text { bhendi yellow vein } \\
\text { alphasatellite }\end{array}$ & FN658716 & BhYVA & IN-Har-07 \\
\hline & & & $\begin{array}{l}\text { Cassava mosaic } \\
\text { Madagascar alphas- } \\
\text { atellite }\end{array}$ & $\begin{array}{l}\text { cassava mosaic } \\
\text { Madagascar alph- } \\
\text { asatellite }\end{array}$ & HE984148 & CMMGA & MG-Diana-635A1-11 \\
\hline & & & $\begin{array}{l}\text { Chilli leaf curl alphas- } \\
\text { atellite }\end{array}$ & $\begin{array}{l}\text { chilli leaf curl alph- } \\
\text { asatellite }\end{array}$ & KF471043 & $\mathrm{ChLCuA}$ & IN-273-06 \\
\hline & & & $\begin{array}{l}\text { Cotton leaf curl Egypt } \\
\text { alphasatellite }\end{array}$ & $\begin{array}{l}\text { cotton leaf curl } \\
\text { Egypt alphasatel- } \\
\text { lite }\end{array}$ & AJ512960 & CLCuEA & EG-SB45-95 \\
\hline & & & $\begin{array}{l}\text { Cotton leaf curl } \\
\quad \text { Gezira alphasatellite }\end{array}$ & $\begin{array}{l}\text { cotton leaf curl } \\
\text { Gezira alphasat- } \\
\text { ellite }\end{array}$ & EU589450 & CLCuGeA & ML-Bamako-okra-06 \\
\hline & & & $\begin{array}{l}\text { Cotton leaf curl Luc- } \\
\text { know alphasatellite }\end{array}$ & $\begin{array}{l}\text { cotton leaf curl } \\
\text { Lucknow alphas- } \\
\text { atellite }\end{array}$ & HQ343234 & CLCuLuA & IN-Luc-10 \\
\hline & & 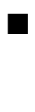 & $\begin{array}{l}\text { Cotton leaf curl Mul- } \\
\text { tan alphasatellite }\end{array}$ & $\begin{array}{l}\text { cotton leaf curl } \\
\text { Multan alphasat- } \\
\text { ellite }\end{array}$ & AJ132344 & CLCuMuA & PK-Fai01-98 \\
\hline
\end{tabular}


Table 1 (continued)

\begin{tabular}{|c|c|c|c|c|c|c|}
\hline Subfamily & Genus & Species & Alphasatellite & Accession & $\begin{array}{l}\text { Alphasatellite } \\
\text { acronym }\end{array}$ & Isolate ID \\
\hline & & $\begin{array}{l}\text { Gossypium darwinii } \\
\text { symptomless alphas- } \\
\text { atellite }\end{array}$ & $\begin{array}{l}\text { Gossypium darwinii } \\
\text { symptomless } \\
\text { alphasatellite }\end{array}$ & EU384623 & GDarSLA & PK-Mul-Dav7C-06 \\
\hline & & $\begin{array}{l}\text { Malvastrum yellow } \\
\text { mosaic alphasatel- } \\
\text { lite }\end{array}$ & $\begin{array}{l}\text { malvastrum yellow } \\
\text { mosaic alphasat- } \\
\text { ellite }\end{array}$ & AM236765 & MaYA & $\mathrm{CN}-\mathrm{Hn} 39$ \\
\hline & & $\begin{array}{l}\text { Malvastrum yellow } \\
\text { mosaic Cameroon } \\
\text { alphasatellite }\end{array}$ & $\begin{array}{l}\text { malvastrum yellow } \\
\text { mosaic Cameroon } \\
\text { alphasatellite }\end{array}$ & FN675297 & MaYMCMA & $\begin{array}{l}\text { CM-Mundemba- } \\
\text { UMU1D1-08 }\end{array}$ \\
\hline & & $\begin{array}{l}\text { Pedilanthus leaf curl } \\
\text { alphasatellite }\end{array}$ & $\begin{array}{l}\text { pedilanthus leaf curl } \\
\text { alphasatellite }\end{array}$ & KX168428 & PeLCuA & IN-carrot \\
\hline & & $\begin{array}{l}\text { Sida leaf curl alphas- } \\
\text { atellite }\end{array}$ & $\begin{array}{l}\text { sida leaf curl alph- } \\
\text { asatellite }\end{array}$ & FR772088 & SiLCuA & $\begin{array}{l}\text { PK-Lahore-Alcea } \\
\text { rosea-06 }\end{array}$ \\
\hline & & $\begin{array}{l}\text { Sida yellow vein Viet- } \\
\text { nam alphasatellite }\end{array}$ & $\begin{array}{l}\text { sida yellow vein } \\
\text { Vietnam alphas- } \\
\text { atellite }\end{array}$ & DQ641718 & SiYVVA & VN-Han-05 \\
\hline & & $\begin{array}{l}\text { Sunflower leaf curl } \\
\text { Karnataka alphas- } \\
\text { atellite }\end{array}$ & $\begin{array}{l}\text { sunflower leaf curl } \\
\text { Karnataka alphas- } \\
\text { atellite }\end{array}$ & JX569789 & SLCuKaA & IN-tomato-11 \\
\hline & & $\begin{array}{l}\text { Synedrella leaf curl } \\
\text { alphasatellite }\end{array}$ & $\begin{array}{l}\text { synedrella leaf curl } \\
\text { alphasatellite }\end{array}$ & KJ939346 & SyLCuA & IN-Por-synf_1-09 \\
\hline & & $\begin{array}{l}\text { Tobacco curly shoot } \\
\text { alphasatellite }\end{array}$ & $\begin{array}{l}\text { tobacco curly shoot } \\
\text { alphasatellite }\end{array}$ & HQ407396 & TbCSA & $\begin{array}{l}\text { IN-WSF1-Helian- } \\
\text { thus-10 }\end{array}$ \\
\hline & & $\begin{array}{l}\text { Tomato leaf curl Buea } \\
\text { alphasatellite }\end{array}$ & $\begin{array}{l}\text { tomato leaf curl } \\
\text { Buea alphasatel- } \\
\text { lite }\end{array}$ & FN675299 & ToLCuBuA & $\begin{array}{l}\text { CM-Buea- } \\
\text { TOS2D1-07 }\end{array}$ \\
\hline & & $\begin{array}{l}\text { Tomato leaf curl Cam- } \\
\text { eroon alphasatellite }\end{array}$ & $\begin{array}{l}\text { tomato leaf curl } \\
\text { Cameroon alphas- } \\
\text { atellite }\end{array}$ & FN675296 & ToLCuCMA & $\begin{array}{l}\text { CM-Buea- } \\
\text { OMHD3-08 }\end{array}$ \\
\hline & & $\begin{array}{l}\text { Tomato yellow leaf } \\
\text { curl China alphas- } \\
\text { atellite }\end{array}$ & $\begin{array}{l}\text { tomato yellow leaf } \\
\text { curl China alphas- } \\
\text { atellite }\end{array}$ & AM749493 & TYLCuCA & PK-Fai-09 \\
\hline & & $\begin{array}{l}\text { Tomato yellow leaf } \\
\text { curl Thailand alph- } \\
\text { asatellite }\end{array}$ & $\begin{array}{l}\text { tomato yellow leaf } \\
\text { curl Thailand } \\
\text { alphasatellite }\end{array}$ & AJ579359 & TYLCuTHA & TH-Y70-03 \\
\hline & & $\begin{array}{l}\text { Tomato yellow leaf } \\
\text { curl Yunnan alphas- } \\
\text { atellite }\end{array}$ & $\begin{array}{l}\text { tomato yellow leaf } \\
\text { curl Yunnan alph- } \\
\text { asatellite }\end{array}$ & KX759649 & TYLCuYA & CN-YN4368_69-14 \\
\hline & Gosmusatellite & $\begin{array}{l}\text { Gossypium muste- } \\
\text { linum symptomless } \\
\text { alphasatellite }\end{array}$ & $\begin{array}{l}\text { Gossypium musteli- } \\
\text { num symptomless } \\
\text { alphasatellite }\end{array}$ & EU384656 & GMusSLA & PK-Mul-2-06 \\
\hline & & $\begin{array}{l}\text { Hollyhock yellow vein } \\
\text { alphasatellite }\end{array}$ & $\begin{array}{l}\text { hollyhock yellow } \\
\text { vein alphasatellite }\end{array}$ & FR772086 & HoYVA & PK-Lahor-17_5-06 \\
\hline & & $\begin{array}{l}\text { Mesta yellow vein } \\
\text { mosaic alphasatel- } \\
\text { lite }\end{array}$ & $\begin{array}{l}\text { mesta yellow vein } \\
\text { mosaic alphasat- } \\
\text { ellite }\end{array}$ & JX183090 & MeYVMA & IN-Ludhiana-Okra-10 \\
\hline & & $\begin{array}{l}\text { Okra enation leaf curl } \\
\text { alphasatellite }\end{array}$ & $\begin{array}{l}\text { okra enation leaf } \\
\text { curl alphasatellite }\end{array}$ & HF546575 & OEnLCuA & IN-Surat-11 \\
\hline & & $\begin{array}{l}\text { Okra yellow crinkle } \\
\text { Cameroon alphas- } \\
\text { atellite }\end{array}$ & $\begin{array}{l}\text { okra yellow crinkle } \\
\text { Cameroon alphas- } \\
\text { atellite }\end{array}$ & FN675285 & OkYCCA & CM-Lys1sp3-08 \\
\hline & & $\begin{array}{l}\text { Vernonia yellow vein } \\
\text { Fujian alphasatellite }\end{array}$ & $\begin{array}{l}\text { vernonia yellow } \\
\text { vein Fujian alph- } \\
\text { asatellite }\end{array}$ & КС959931 & VeYVFA & $\begin{array}{l}\text { IN-Vamban-black- } \\
\text { gram-12 }\end{array}$ \\
\hline & Unassigned & $\begin{array}{l}\text { Dragonfly associated } \\
\text { alphasatellite }\end{array}$ & $\begin{array}{l}\text { dragonfly associated } \\
\text { alphasatellite }\end{array}$ & JX458742 & DfaA & PR-09 \\
\hline
\end{tabular}


Table 1 (continued)

\begin{tabular}{|c|c|c|c|c|c|c|}
\hline Subfamily & Genus & Species & Alphasatellite & Accession & $\begin{array}{l}\text { Alphasatellite } \\
\text { acronym }\end{array}$ & Isolate ID \\
\hline & & $\begin{array}{l}\text { Whitefly associated } \\
\text { Guatemala alphasat- } \\
\text { ellite } 1\end{array}$ & $\begin{array}{l}\text { whitefly associated } \\
\text { Guatemala alphas- } \\
\text { atellite } 1\end{array}$ & KT099172 & WfaGA 1 & GT-GtTo2_2-12 \\
\hline \multirow[t]{19}{*}{$\begin{array}{l}\text { Nanoalphasatel- } \\
\text { litinae }\end{array}$} & Babusatellite & $\begin{array}{l}\text { Banana bunchy top } \\
\text { alphasatellite } 2\end{array}$ & $\begin{array}{l}\text { banana bunchy top } \\
\text { alphasatellite } 2\end{array}$ & AF416471 & BBTA 2 & VN-VB-sat3-00 \\
\hline & & $\begin{array}{c}\text { Banana bunchy top } \\
\text { alphasatellite } 1\end{array}$ & $\begin{array}{l}\text { banana bunchy top } \\
\text { alphasatellite } 1\end{array}$ & L32167 & BBTA 1 & 93 \\
\hline & & $\begin{array}{l}\text { Banana bunchy top } \\
\text { alphasatellite } 3\end{array}$ & $\begin{array}{l}\text { banana bunchy top } \\
\text { alphasatellite } 3\end{array}$ & HQ616080 & BBTA 3 & CN-Haikou-10 \\
\hline & & $\begin{array}{l}\text { Cardamom bushy } \\
\text { dwarf alphasatellite }\end{array}$ & $\begin{array}{l}\text { cardamom bushy } \\
\text { dwarf alphasatel- } \\
\text { lite }\end{array}$ & KF435148 & $\mathrm{CaBuDA}$ & IN-Kalimpong-07 \\
\hline & Clostunsatellite & $\begin{array}{r}\text { Milk vetch dwarf } \\
\text { alphasatellite } 2\end{array}$ & $\begin{array}{r}\text { milk vetch dwarf } \\
\text { alphasatellite } 2\end{array}$ & AB000922 & MVDA 2 & JR-C3-96 \\
\hline & & $\begin{array}{l}\text { Pea necrotic yellow } \\
\text { dwarf alphasatel- } \\
\text { lite } 2\end{array}$ & $\begin{array}{l}\text { pea necrotic yellow } \\
\text { dwarf alphasatel- } \\
\text { lite } 2\end{array}$ & KC979052 & PNYDA 2 & $\begin{array}{l}\text { AT-Gross_Enzers- } \\
\text { dorf_1-10 }\end{array}$ \\
\hline & & $\begin{array}{l}\text { Sophora yellow stunt } \\
\text { alphasatellite } 4\end{array}$ & $\begin{array}{l}\text { sophora yellow stunt } \\
\text { alphasatellite } 4\end{array}$ & KX534409 & SYSA 4 & IR-Kerman-Ta1_9-14 \\
\hline & & $\begin{array}{l}\text { Sophora yellow stunt } \\
\text { alphasatellite } 5\end{array}$ & $\begin{array}{l}\text { sophora yellow stunt } \\
\text { alphasatellite } 5\end{array}$ & KX534398 & SYSA 5 & IR-Kerman-Ta1_8-14 \\
\hline & & $\begin{array}{l}\text { Subterranean clover } \\
\text { stunt alphasatel- } \\
\text { lite } 2\end{array}$ & $\begin{array}{l}\text { subterranean clover } \\
\text { stunt alphasatel- } \\
\text { lite } 2\end{array}$ & U16735 & SCSA 2 & AU-C6-93 \\
\hline & Fabenesatellite & $\begin{array}{l}\text { Faba bean necrotic } \\
\text { yellows alphasatel- } \\
\text { lite } 2\end{array}$ & $\begin{array}{l}\text { faba bean necrotic } \\
\text { yellows alphasat- } \\
\text { ellite } 2\end{array}$ & AJ005966 & FBNYA 2 & SY-C9-93 \\
\hline & Milvetsatellite & $\begin{array}{r}\text { Milk vetch dwarf } \\
\text { alphasatellite } 3\end{array}$ & $\begin{array}{r}\text { milk vetch dwarf } \\
\text { alphasatellite } 3\end{array}$ & AB009047 & MVDA 3 & JR-C10-97 \\
\hline & $\begin{array}{l}\text { Mivedwarsatel- } \\
\text { lite }\end{array}$ & $\begin{array}{l}\text { Faba bean necrotic } \\
\text { stunt alphasatellite }\end{array}$ & $\begin{array}{l}\text { faba bean necrotic } \\
\text { stunt alphasatellite }\end{array}$ & KC978990 & FBNSA & AZ-12b-10 \\
\hline & & $\begin{array}{r}\text { Milk vetch dwarf } \\
\text { alphasatellite } 1\end{array}$ & $\begin{array}{r}\text { milk vetch dwarf } \\
\text { alphasatellite } 1\end{array}$ & AB000920 & MVDA 1 & JR-C1-96 \\
\hline & & $\begin{array}{l}\text { Pea necrotic yellow } \\
\text { dwarf alphasatel- } \\
\text { lite } 1\end{array}$ & $\begin{array}{l}\text { pea necrotic yellow } \\
\text { dwarf alphasatel- } \\
\text { lite } 1\end{array}$ & КC979051 & PNYDA 1 & $\begin{array}{l}\text { AT-Gross_Enzers- } \\
\text { dorf_1-10 }\end{array}$ \\
\hline & & $\begin{array}{l}\text { Sophora yellow stunt } \\
\text { alphasatellite } 2\end{array}$ & $\begin{array}{l}\text { sophora yellow stunt } \\
\text { alphasatellite } 2\end{array}$ & KX534408 & SYSA 2 & IR-Kerman-Ta1_4-14 \\
\hline & Sophoyesatellite & $\begin{array}{c}\text { Sophora yellow stunt } \\
\text { alphasatellite } 3\end{array}$ & $\begin{array}{l}\text { sophora yellow stunt } \\
\text { alphasatellite } 3\end{array}$ & KX534400 & SYSA 3 & $\begin{array}{l}\text { IR-Kerman- } \\
\text { Ta1_7a-14 }\end{array}$ \\
\hline & Subclovsatellite & $\begin{array}{l}\text { Faba bean necrotic } \\
\text { yellows alphasatel- } \\
\text { lite } 1\end{array}$ & $\begin{array}{l}\text { faba bean necrotic } \\
\text { yellows alphasat- } \\
\text { ellite } 1\end{array}$ & X80879 & FBNYA 1 & SY-C1-88 \\
\hline & & $\begin{array}{l}\text { Sophora yellow stunt } \\
\text { alphasatellite } 1\end{array}$ & $\begin{array}{l}\text { sophora yellow stunt } \\
\text { alphasatellite } 1\end{array}$ & KX534399 & SYSA 1 & $\begin{array}{l}\text { IR-Kerman- } \\
\text { Ta1_1a-14 }\end{array}$ \\
\hline & & $\begin{array}{l}\text { Subterranean clover } \\
\text { stunt alphasatel- } \\
\text { lite } 1\end{array}$ & $\begin{array}{l}\text { subterranean clover } \\
\text { stunt alphasatel- } \\
\text { lite } 1\end{array}$ & U16731 & SCSCA 1 & AU-C2-93 \\
\hline Unassigned & Unassigned & $\begin{array}{l}\text { Coconut foliar decay } \\
\text { alphasatellite }\end{array}$ & $\begin{array}{l}\text { coconut foliar decay } \\
\text { alphasatellite }\end{array}$ & M29963 & CFDA & 89 \\
\hline
\end{tabular}

Type species

The type species of each genus is in bold font 
identity to any one sequence formerly classified as belonging to that species even if it has $<88 \%$ pairwise identity to all other sequences classified as belonging to that species.

\section{Nanoalphasatellitinae}

Sequences of nanovirus-associated alphasatellites were downloaded from GenBank ( $1^{\text {st }}$ May 2017). These sequences were checked to make sure they were complete and had intact Rep open reading frames. The 54 filtered nanovirusassociated alphasatellites were analysed with SDT v1.2 [29]. The sequecnes were aligned using MUSCLE [9] and the resulting alignment was used to infer a maximum likelihood phylogenetic tree using IQ-TREE [31] with the nucleotide substitution model, TIM2+F+G4 (adjudged the best fitting model for these sequences by ModelFinder [20]). The tree was rooted with representative DNA-R sequences of nanoviruses (AB027511, AF102780, AJ290434, AJ749894, EF546807, GU553134, HE654123, JX867550, KC978949, KC979035, KC979054, KX534388, KX534389, KX534390, KX534391, MF535450).

The distribution of 1326 pairwise identities obtained from every possible pair of the 54 nanovirus-associated alphasatellites had troughs at $\sim 67 \%$ and $~ 80 \%$ (Figure 5), and these were chosen respectively as genus and species demarcation cut offs that would yield the minimum number of classification conflicts (Figure 5). The species demarcation threshold implies the existence of at least 19 nanovirusassociated alphasatellite species and the genus demarcation threshold implies the existence of at least seven genera (Figures 6 and 7). The seven genera and 19 species are supported by the phylogenetic clustering of the nanovirus-associated alphasatellite sequences (Figure 7). A summary of the genera and species in the subfamily Nanoalphasatellitinae is provided in Table 1.

\section{Guidelines for classification of new nanoalphasatellites species}

Pairs of nanoalphasatellite sequences with $\geq 80 \%$ pairwise identity calculated using pairwise MUSCLE alignments with similarities calculated ignoring sites with gaps (such as is implemented in SDT v1.2 [29], should be considered as members of the same species. Those that hare $<80 \%$ pairwise identity can be considered as new species when coupled with good phylogenetic support.

We recommend that the following steps, similar to those outlined for geminiviruses $[6,28,46]$ be taken to resolve such conflicts in cases where 1) a complete nanoalphasatellite sequence shares $\geq 80 \%$ pairwise identity to sequences that have been assigned to two different species; or 2) a complete nanoalphasatellite sequence shares $\geq 80 \%$ genome-wide pairwise identity to one or a few sequences assigned to a particular species, even though it shares $<80 \%$ identity with the majority of sequences in that species.

1) The new nanoalphasatellite molecule sequence should be considered as belonging to the species containing sequences with which it shares the highest percentage pairwise identity.

2) The nanoalphasatellite should be classified as belonging to any species with which it shares $\geq 80 \%$ pairwise identity to any one sequence formerly classified as belonging

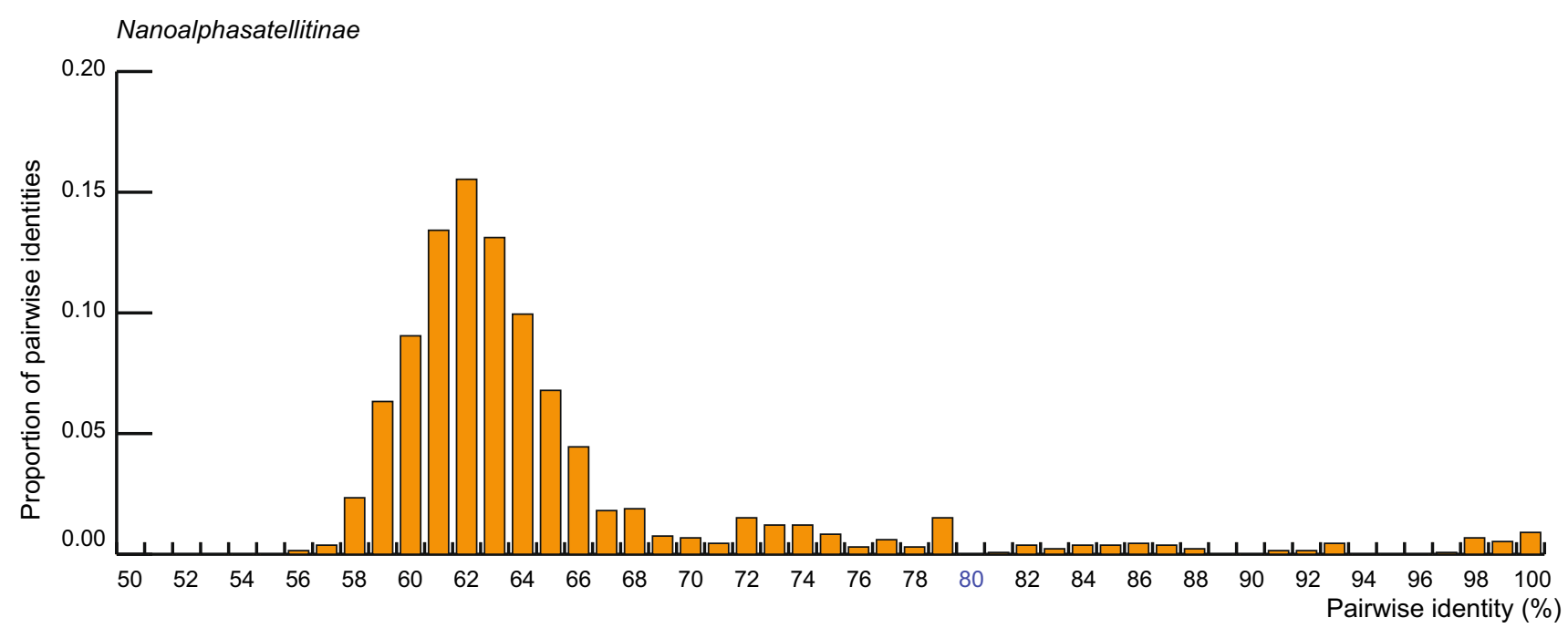

Fig. 5 Distribution of pairwise identities of nanovirus-associated alphasatellites determined using SDT v1.2 [29] 
Fig. 6 A 'three color' pairwise identity matrix inferred using SDT v1.2 [29] showing that both the genera demarcation threshold of $\sim 67 \%$ and that for species at $80 \%$ are supported

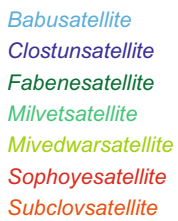

to that species even if it has $<80 \%$ pairwise identity to all other sequences classified as belonging to that species.

\section{Unassigned species in the family Alphasatellitidae}

Sequence M29963 (Table 1; species Coconut foliar decay alphasatellite) was isolated from a coconut palm affected by coconut foliar decay disease [36], a disease earlier shown to be associated with a circular ssDNA virus [14, 33-35]. However, no virus has so far been identified or characterized infecting coconut even though virions have been described [35]. M29963 does not have all the hallmarks of either Geminialphasatellitinae or Nanoalphasatellitinae alphasatellites in that it has a size (1291 nt) intermediate between the alphasatellites assigned to these sub-families and lacks an A-rich sequence. Furthermore, it was isolated from a monocotyledonous host. Two geminialphasatellites have been isolated from monocotyledonous plants and both of these have features typical of the Geminialphasatellitinae [24]; and twelve have been isolated from bananas and cardamom, all of which have similarities to other nanoalphasatellites [2, $11,18,25,53]$. We therefore propose this molecule to represent an unassigned species in the family Alphasatellitidae. It 


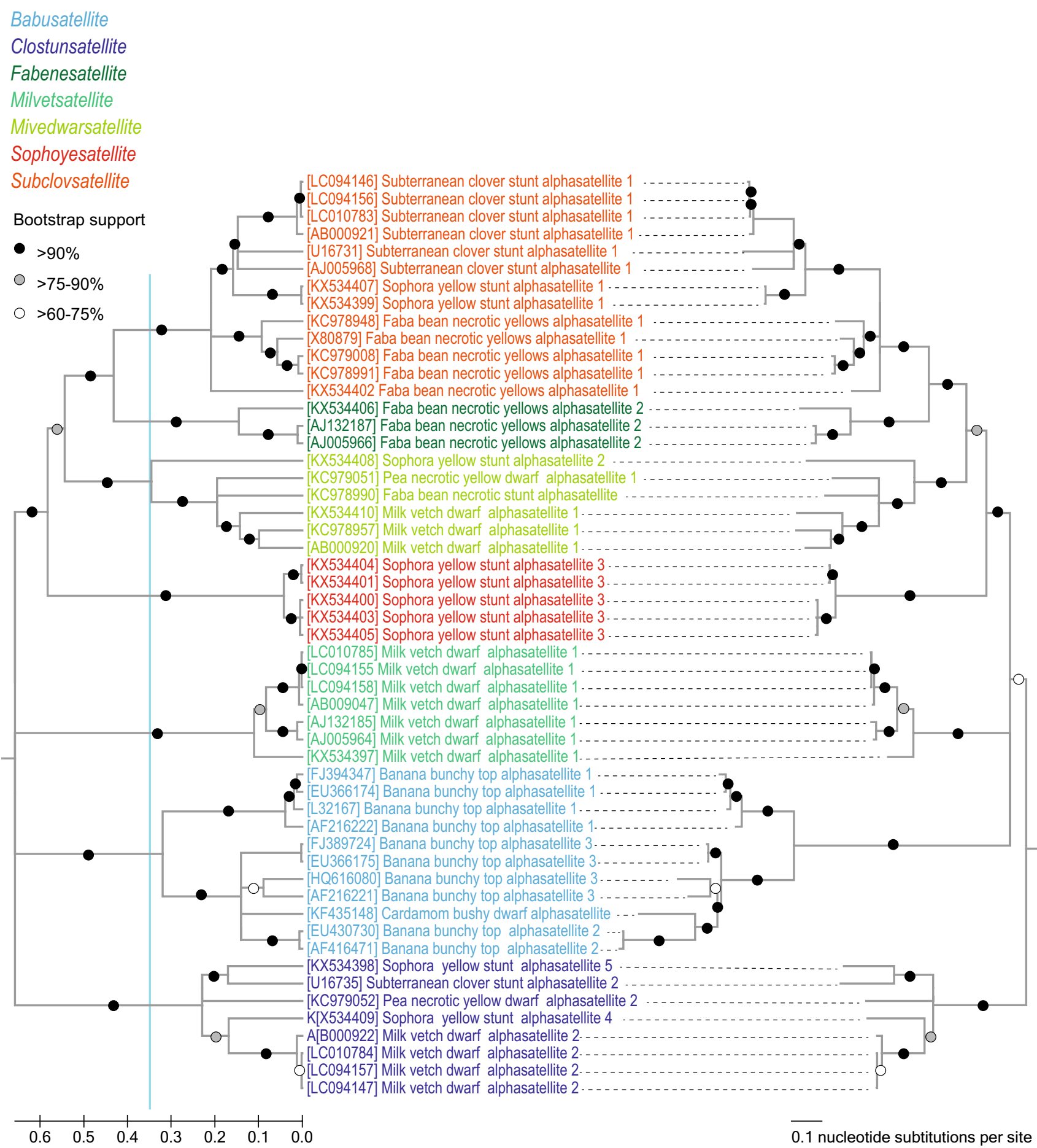

Fig. 7 Maximum likelihood phylogenetic trees of the nanovirusassociated alphasatellite sequences inferred using IQ-TREE [31] with TIM2+F+I+G4 chosen as the best fit model. Branches with less than

is likely that it is a member of a third sub-family that could be formally proposed once more member sequences have been identified.
$60 \%$ bootstrap support have been collapsed. The Maximum likelihood phylogenetic tree on the left is a guide for genera demarcation with the cyan line showing the rough $67 \%$ threshold

\section{Concluding remarks}

The last decade has seen a flurry of activity in the identification of circular ssDNA molecules and viruses primarily 
being driven by multiple displacement amplification techniques coupled with affordable sequencing (both Sanger and high throughput). This has resulted in the identification of a large number of sequences corresponding to diverse viral and viral-like elements sequences from symptomatic and asymptomatic hosts, and from environmental sampling using viral metagenomics approaches. The communication by Simmonds et al. [41] addressed the classification of diverse groups of viral sequences derived from metagenomics approaches and this has resulted in significant changes in viral classification. For example, new families have been established to accommodate virophages and satellite viruses [23] and various novel groups of circular Rep encoding ssDNA viruses derived from environmental sources (with unknown hosts or pathology) [22, 47, 49]. In line with these significant changes on classification of viruses and viral-like elements, we communicate the establishment of a new family Alphasatellitidae with two sub-families Geminialphasatellitinae and Nanoalphasatellitinae for geminivirus- and nanovirus-alphasatellite molecules.

Acknowledgements The authors are grateful to Drs. J. K. Brown and A. Idris for providing unpublished begomovirus-associated alphasatellite sequences for the preliminary analysis.

\section{Compliance with ethical standards}

Conflict of interest The authors declare there are no conflicts of interest.

Research involving human participants and/or animals The research did not involve human participants or animals.

Informed consent The research did not involve human participants or animals.

\section{References}

1. Abbas Q, Amin I, Mansoor S, Shafiq M, Wassenegger M, Briddon RW (2017) The Rep proteins encoded by alphasatellites restore expression of a transcriptionally silenced green fluorescent protein transgene in Nicotiana benthamiana. VirusDisease. https://doi. org/10.1007/s13337-017-0413-5

2. Bell KE, Dale JL, Ha CV, Vu MT, Revill PA (2002) Characterisation of Rep-encoding components associated with banana bunchy top nanovirus in Vietnam. Arch Virol 147:695-707

3. Bottcher B, Unseld S, Ceulemans H, Russell RB, Jeske H (2004) Geminate structures of African cassava mosaic virus. J Virol 78:6758-6765

4. Briddon RW, Bull SE, Amin I, Mansoor S, Bedford ID, Rishi N, Siwatch SS, Zafar Y, Abdel-Salam AM, Markham PG (2004) Diversity of DNA 1: a satellite-like molecule associated with monopartite begomovirus-DNA beta complexes. Virology 324:462-474

5. Brown JK, Fauquet CM, Briddon RW, Zerbini M, Moriones E, Navas-Castillo J (2012) Geminiviridae. In: King AMQ, Adams MJ, Carstens EB, Lefkowitz EJ (eds) Virus taxonomy: ninth report of the International Committee on Taxonomy of Viruse. Elsevier, Amsterdam, pp 351-373

6. Brown JK, Zerbini FM, Navas-Castillo J, Moriones E, RamosSobrinho R, Silva JC, Fiallo-Olive E, Briddon RW, HernandezZepeda C, Idris A, Malathi VG, Martin DP, Rivera-Bustamante R, Ueda S, Varsani A (2015) Revision of Begomovirus taxonomy based on pairwise sequence comparisons. Arch Virol 160:1593-1619

7. Burns TM, Harding RM, Dale JL (1995) The genome organization of banana bunchy top virus: analysis of six ssDNA components. J Gen Virol 76:1471-1482

8. Casado CG, Javier Ortiz G, Padron E, Bean SJ, McKenna R, Agbandje-McKenna M, Boulton MI (2004) Isolation and characterization of subgenomic DNAs encapsidated in "single" $\mathrm{T}=1$ isometric particles of Maize streak virus. Virology 323:164-171

9. Edgar RC (2004) MUSCLE: multiple sequence alignment with high accuracy and high throughput. Nucleic Acids Res 32:1792-1797

10. Ferro CG, Silva JP, Xavier CAD, Godinho MT, Lima ATM, Mar TB, Lau D, Zerbini FM (2017) The ever increasing diversity of begomoviruses infecting non-cultivated hosts: new species from Sida spp. and Leonurus sibiricus, plus two New World alphasatellites. Ann Appl Biol 170:204-218

11. Fu HC, Hu JM, Hung TH, Su HJ, Yeh HH (2009) Unusual events involved in Banana bunchy top virus strain evolution. Phytopathology 99:812-822

12. Gallet R, Kraberger S, Filloux D, Galzi S, Fontes H, Martin DP, Varsani A, Roumagnac P (2018) Nanovirus-alphasatellite complex identified in Vicia cracca in the Rhone delta region of France. Arch Virol 163:695-700

13. Hafner GJ, Stafford MR, Wolter LC, Harding RM, Dale JL (1997) Nicking and joining activity of banana bunchy top virus replication protein in vitro. J Gen Virol 78:1795-1799

14. Hanold D, Langridge P, Randles JW (1988) The use of cloned sequences for the Identification of coconut foliar decay diseaseassociated DNA. J Gen Virol 69:1323-1329

15. Harding RM, Burns TM, Hafner G, Dietzgen RG, Dale JL (1993) Nucleotide sequence of one component of the banana bunchy top virus genome contains a putative replicase gene. J Gen Virol 74:323-328

16. Heydarnejad J, Kamali M, Massumi H, Kvarnheden A, Male MF, Kraberger S, Stainton D, Martin DP, Varsani A (2017) Identification of a nanovirus-alphasatellite complex in Sophora alopecuroides. Virus Res 235:24-32

17. Hipp K, Grimm C, Jeske H, Bottcher B (2017) Near-atomic resolution structure of a plant geminivirus determined by electron cryomicroscopy. Structure 25(1303-1309):e1303

18. Horser C, Harding R, Dale J (2001) Banana bunchy top nanovirus DNA-1 encodes the 'master' replication initiation protein. J Gen Virol 82:459-464

19. Idris AM, Shahid MS, Briddon RW, Khan AJ, Zhu JK, Brown JK (2011) An unusual alphasatellite associated with monopartite begomoviruses attenuates symptoms and reduces betasatellite accumulation. J Gen Virol 92:706-717

20. Kalyaanamoorthy S, Minh BQ, Wong TKF, von Haeseler A, Jermiin LS (2017) ModelFinder: fast model selection for accurate phylogenetic estimates. Nat Methods 14:587-589

21. Kon T, Rojas MR, Abdourhamane IK, Gilbertson RL (2009) Roles and interactions of begomoviruses and satellite DNAs associated with okra leaf curl disease in Mali, West Africa. J Gen Virol 90:1001-1013

22. Krupovic M, Ghabrial SA, Jiang D, Varsani A (2016) Genomoviridae: a new family of widespread single-stranded DNA viruses. Arch Virol 161:2633-2643

23. Krupovic M, Kuhn JH, Fischer MG (2016) A classification system for virophages and satellite viruses. Arch Virol 161:233-247 
24. Kumar J, Kumar J, Singh SP, Tuli R (2014) Association of satellites with a mastrevirus in natural infection: complexity of wheat dwarf India virus disease. J Virol 88:7093-7104

25. Mandal B, Shilpi S, Barman AR, Mandal S, Varma A (2013) Nine novel DNA components associated with the foorkey disease of large cardamom: evidence of a distinct babuvirus species in Nanoviridae. Virus Res 178:297-305

26. Mansoor S, Khan SH, Bashir A, Saeed M, Zafar Y, Malik KA, Briddon R, Stanley J, Markham PG (1999) Identification of a novel circular single-stranded DNA associated with cotton leaf curl disease in Pakistan. Virology 259:190-199

27. Mar TB, Mendes IR, Lau D, Fiallo-Olive E, Navas-Castillo J, Alves MS, Murilo Zerbini F (2017) Interaction between the New World begomovirus Euphorbia yellow mosaic virus and its associated alphasatellite: effects on infection and transmission by the whitefly Bemisia tabaci. J Gen Virol 98:1552-1562

28. Muhire B, Martin DP, Brown JK, Navas-Castillo J, Moriones E, Zerbini FM, Rivera-Bustamante R, Malathi VG, Briddon RW, Varsani A (2013) A genome-wide pairwise-identity-based proposal for the classification of viruses in the genus Mastrevirus (family Geminiviridae). Arch Virol 158:1411-1424

29. Muhire BM, Varsani A, Martin DP (2014) SDT: a virus classification tool based on pairwise sequence alignment and identity calculation. PLoS One 9:e108277

30. Nawaz-Ul-Rehman MS, Nahid N, Mansoor S, Briddon RW, Fauquet CM (2010) Post-transcriptional gene silencing suppressor activity of two non-pathogenic alphasatellites associated with a begomovirus. Virology 405:300-308

31. Nguyen LT, Schmidt HA, von Haeseler A, Minh BQ (2015) IQTREE: a fast and effective stochastic algorithm for estimating maximum-likelihood phylogenies. Mol Biol Evol 32:268-274

32. Paprotka T, Metzler V, Jeske H (2010) The first DNA 1-like alpha satellites in association with New World begomoviruses in natural infections. Virology 404:148-157

33. Randles JW (1986) Association of single-stranded DNA with the foliar decay disease of coconut palm in Vanuatu. Phytopathology 76:889-894

34. Randles JW, Hanold D, Julia JF (1987) Small circular singlestranded DNA associated with foliar decay disease of coconut palm in Vanuatu. J Gen Virol 68:273-280

35. Randles JW, Hanold D (1989) Coconut foliar decay virus particles are 20-nm icosahedra. Intervirology 30:177-180

36. Rohde W, Randles JW, Langridge P, Hanold D (1990) Nucleotide sequence of a circular single-stranded DNA associated with coconut foliar decay virus. Virology 176:648-651

37. Romay G, Chirinos D, Geraud-Pouey F, Desbiez C (2010) Association of an atypical alphasatellite with a bipartite New World begomovirus. Arch Virol 155:1843-1847

38. Sano Y, Wada M, Hashimoto Y, Matsumoto T, Kojima M (1998) Sequences of ten circular ssDNA components associated with the milk vetch dwarf virus genome. J Gen Virol 79:3111-3118

39. Sattar MN, Kvarnheden A, Saeed M, Briddon RW (2013) Cotton leaf curl disease-an emerging threat to cotton production worldwide. J Gen Virol 94:695-710

40. Saunders K, Stanley J (1999) A nanovirus-like DNA component associated with yellow vein disease of Ageratum conyzoides: evidence for interfamilial recombination between plant DNA viruses. Virology 264:142-152

41. Simmonds P, Adams MJ, Benko M, Breitbart M, Brister JR, Carstens EB, Davison AJ, Delwart E, Gorbalenya AE, Harrach B, Hull R, King AM, Koonin EV, Krupovic M, Kuhn JH, Lefkowitz EJ, Nibert ML, Orton R, Roossinck MJ, Sabanadzovic S, Sullivan
MB, Suttle CA, Tesh RB, van der Vlugt RA, Varsani A, Zerbini FM (2017) Consensus statement: virus taxonomy in the age of metagenomics. Nat Rev Microbiol 15:161-168

42. Stainton D, Martin DP, Muhire BM, Lolohea S, Halafihi M, Lepoint P, Blomme G, Crew KS, Sharman M, Kraberger S, Dayaram A, Walters M, Collings DA, Mabvakure B, Lemey P, Harkins GW, Thomas JE, Varsani A (2015) The global distribution of Banana bunchy top virus reveals little evidence for frequent recent, human-mediated long distance dispersal events. Virus Evol 1:vev009

43. Timchenko T, de Kouchkovsky F, Katul L, David C, Vetten HJ, Gronenborn B (1999) A single rep protein initiates replication of multiple genome components of faba bean necrotic yellows virus, a single-stranded DNA virus of plants. J Virol 73:10173-10182

44. Timchenko T, Katul L, Sano Y, de Kouchkovsky F, Vetten HJ, Gronenborn B (2000) The master rep concept in nanovirus replication: identification of missing genome components and potential for natural genetic reassortment. Virology 274:189-195

45. Timchenko T, Katul L, Aronson M, Vega-Arreguin JC, Ramirez BC, Vetten HJ, Gronenborn B (2006) Infectivity of nanovirus DNAs: induction of disease by cloned genome components of Faba bean necrotic yellows virus. J Gen Virol 87:1735-1743

46. Varsani A, Navas-Castillo J, Moriones E, Hernandez-Zepeda C, Idris A, Brown JK, Murilo Zerbini F, Martin DP (2014) Establishment of three new genera in the family Geminiviridae: Becurtovirus, Eragrovirus and Turncurtovirus. Arch Virol 159:2193-2203

47. Varsani A, Krupovic M (2017) Sequence-based taxonomic framework for the classification of uncultured single-stranded DNA viruses of the family Genomoviridae. Virus Evol 3:vew037

48. Varsani A, Roumagnac P, Fuchs M, Navas-Castillo J, Moriones E, Idris A, Briddon RW, Rivera-Bustamante R, Murilo Zerbini F, Martin DP (2017) Capulavirus and Grablovirus: two new genera in the family Geminiviridae. Arch Virol 162:1819-1831

49. Varsani A, Krupovic M (2018) Smacoviridae: a new family of animal-associated single-stranded DNA viruses. Arch Virol. https ://doi.org/10.1007/s00705-018-3820-z

50. Vetten HJ (2008) Nanoviruses. In: Mahy BWJ, Van Regenmortel M (eds) Encyclopedia of virology. Elsevier, Oxford

51. Vetten HJ, Dale JL, Grigoras I, Gronenborn B, Harding R, Randles JW, Sano Y, Thomas JE, Timchenko T, Yeh HH (2012) Nanoviridae. In: King AMQ, Adams MJ, Carstens EB, Lefkowitz EJ (eds) Virus taxonomy: ninth report of the International Committee on Taxonomy of Viruse. Elsevier, Amsterdam, pp 395-404

52. Xie Y, Wu P, Liu P, Gong H, Zhou X (2010) Characterization of alphasatellites associated with monopartite begomovirus/betasatellite complexes in Yunnan, China. Virol J 7:178

53. Yu NT, Zhang YL, Feng TC, Wang JH, Kulye M, Yang WJ, Lin ZS, Xiong Z, Liu ZX (2012) Cloning and sequence analysis of two banana bunchy top virus genomes in Hainan. Virus Genes $44: 488-494$

54. Zerbini FM, Briddon RW, Idris A, Martin DP, Moriones E, NavasCastillo J, Rivera-Bustamante R, Roumagnac P, Varsani A, Ictv Report C (2017) ICTV virus taxonomy profile: Geminiviridae. J Gen Virol 98:131-133

55. Zhang W, Olson NH, Baker TS, Faulkner L, Agbandje-McKenna M, Boulton MI, Davies JW, McKenna R (2001) Structure of the Maize streak virus geminate particle. Virology 279:471-477

56. Zhou X (2013) Advances in understanding begomovirus satellites. Annu Rev Phytopathol 51:357-381 


\section{Affiliations}

\section{Rob W. Briddon ${ }^{1}$. Darren P. Martin ${ }^{2} \cdot$ Philippe Roumagnac $^{3,4}$. Jesús Navas-Castillo ${ }^{5}$ Elvira Fiallo-Olivé ${ }^{5}$. Enrique Moriones ${ }^{5} \cdot$ Jean-Michel Lett $^{6} \cdot$ F. Murilo Zerbini ${ }^{7} \cdot$ Arvind Varsani $^{8,9}{ }^{(1)}$}

$1 \quad$ National Institute for Biotechnology and Genetic Engineering (NIBGE), Faisalabad, Pakistan

2 Computational Biology Group, Faculty of Health Sciences, Institute of Infectious Disease and Molecular Medicine,

University of Cape Town, Observatory, Cape Town 7925, South Africa

3 CIRAD, BGPI, Montpellier, France

4 BGPI, INRA, CIRAD, SupAgro, Univ Montpellier, Montpellier, France

5 Instituto de Hortofruticultura Subtropical y Mediterránea "La Mayora”, Universidad de Málaga-Consejo Superior de Investigaciones Científicas (IHSM-UMA-CSIC),

Algarrobo-Costa, 29750 Málaga, Spain
6 Pôle de Protection des Plantes, CIRAD, UMR PVBMT, 7 chemin de l'IRAT, 97410 Saint-Pierre, Ile de la Réunion, France

7 Dep. de Fitopatologia/Bioagro, Universidade Federal de Viçosa, Viçosa, Minas Gerais 36570-900, Brazil

8 The Biodesign Center for Fundamental and Applied Microbiomics, Center for Evolution and Medicine and School of Life sciences, Arizona State University, Tempe, AZ 85287, USA

9 Structural Biology Research Unit, Department of Integrative Biomedical Sciences, University of Cape Town, Rondebosch, Cape Town 7701, South Africa 